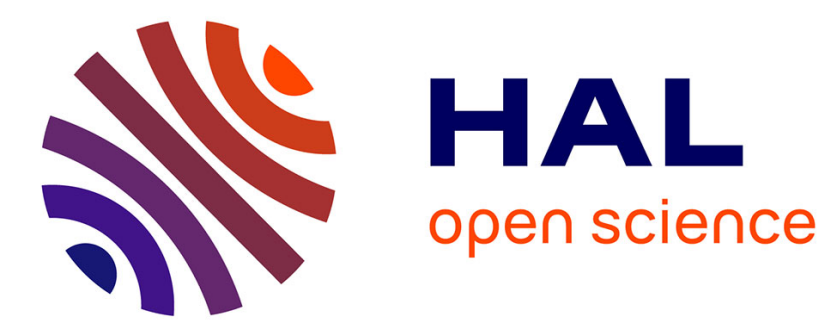

\title{
La mission archéologique française en Dominique
}

Benoît Bérard

\section{To cite this version:}

Benoît Bérard. La mission archéologique française en Dominique. Les Nouvelles de l'archéologie, 2008, Des mers de glace à la Terre de feu (111-112), pp.95-100. hal-00967105

\section{HAL Id: hal-00967105 https://hal.univ-antilles.fr/hal-00967105}

Submitted on 28 Mar 2014

HAL is a multi-disciplinary open access archive for the deposit and dissemination of scientific research documents, whether they are published or not. The documents may come from teaching and research institutions in France or abroad, or from public or private research centers.
L'archive ouverte pluridisciplinaire HAL, est destinée au dépôt et à la diffusion de documents scientifiques de niveau recherche, publiés ou non, émanant des établissements d'enseignement et de recherche français ou étrangers, des laboratoires publics ou privés. 


\title{
La mission archéologique française en Dominique
}

\author{
Benoît Bérard*
}

* Maître de conférences à l'université des Antilles et de la Guyane, EA929 "Archéologie industrielle, histoire et patrimoine de la Caraïbe", chercheur associé de UMR 8096 du CNRS "Archéologie des Amériques", benoit.berard@wanadoo.fr.
$\mathrm{L}$ 'archipel antillais comprend (à l'exception de la Guyane) les seuls territoires français des Amériques. Cependant, même au sein de ces territoires (la Martinique et l'archipel de Guadeloupe), l'archéologie professionnelle ne s'y est vraiment ancrée que dans le dernier quart du $\mathrm{xx}^{\mathrm{e}}$ siècle. C'est à partir de ces "têtes de ponts" que commencent à se développer depuis quelques années des actions en direction d'autres îles de l'archipel. Ainsi, depuis la Martinique, des projets ponctuels ont été développés au début des années 1990 à Trinidad ou à Cuba avec le soutien du ministère français des Affaires étrangères (Rodriguez-Loubet 1995). Le premier programme pluriannuel a été la mission archéologique "Sud-Dominique», mise en place en 2005.

L'expérience qui en est issue permet de tenter un premier bilan tant au niveau des résultats scientifiques qu'en matière de gestion du patrimoine archéologique à la Dominique et, plus largement, au sein des Petites Antilles anglophones. Nous présenterons les difficultés inhérentes à la mise en place d'un programme archéologique dans de petits États indépendants depuis peu qui ne disposent pas d'un outil législatif adéquat. Nous tenterons surtout de montrer l'intérêt qu'il y a, pour les chercheurs français, à sortir de leurs bastions martiniquais et guadeloupéens et nous envisagerons quelques perspectives concernant l'avenir de la recherche archéologique française au sein de la Caraïbe. Elle semble maintenant disposer d'un certain nombre d'atouts qui devraient lui permettre d'y jouer un rôle majeur.

\section{L'archéologie en Dominique}

La Dominique est une île de $751 \mathrm{~km}^{2}$ située au cœur des Petites Antilles. Elle est entourée au sud par la Martinique et au nord par les îles de l'archipel de la Guadeloupe (fig. 1). Occupée pendant au moins deux millénaires par des populations amérindiennes, elle a fait l'objet d'une première colonisation française informelle avant de devenir colonie britannique en 1763. Elle le restera jusqu'en 1978, date où elle acquière son indépendance (Honychurch 1995). Elle est membre de l'Organisation of the Eastern Caribbean States (OECS) depuis 1980, qui regroupe les anciennes colonies britanniques, devenues indépendantes, de la Grenade, Antigua et Barbuda, Saint-Vincent et les Grenadines, Sainte-Lucie, la Dominique et Saint Christophe et Nevis. La Dominique compte environ 70000 habitants dont 3000 , regroupés au sein du territoire caraïbe, constituent la dernière communauté amérindienne des Antilles.

La recherche archéologique en Dominique s'est limitée jusqu'à ce jour à l'étude du peuplement précolombien de l'île. Et même en ce domaine, il s'agit d'une des îles les plus pauvrement documentées des Antilles. Avant notre arrivée, sept articles seulement avaient été publiés sur ce sujet et tous dataient de plus de trente ans. Le premier est celui de W. Fewkes, qui ne resta que quelques heures dans l'île et en profita pour jeter un coup d'œil aux objets lithiques conservés à la bibliothèque de Roseau (Fewkes 1922). Après lui, M. McKusick réalisa les premiers sondages archéologiques dans le site de Vieille Case (McKusick 1960). Cependant, la contribution ayant eu le plus grand impact sur l'archéologie de la Dominique fut l'article présenté par C. Evans lors du second congrès pour l'étude des civilisations précolombiennes des Petites Antilles (Evans 1968). Dans "The lack of archaeology in Dominica", il explique que la très faible quantité de sites amérindiens qu'il a été capable de découvrir est un reflet fidèle de la réalité de l'occupation précolombienne de l'île. Pour justifier cette position, il développe l'hypothèse selon laquelle les ressources limitées disponibles en Dominique (entre autres du fait de l'absence de mangroves) n'y ont pas permis le maintien d'une importante population. Cet article, même si quelques chercheurs 


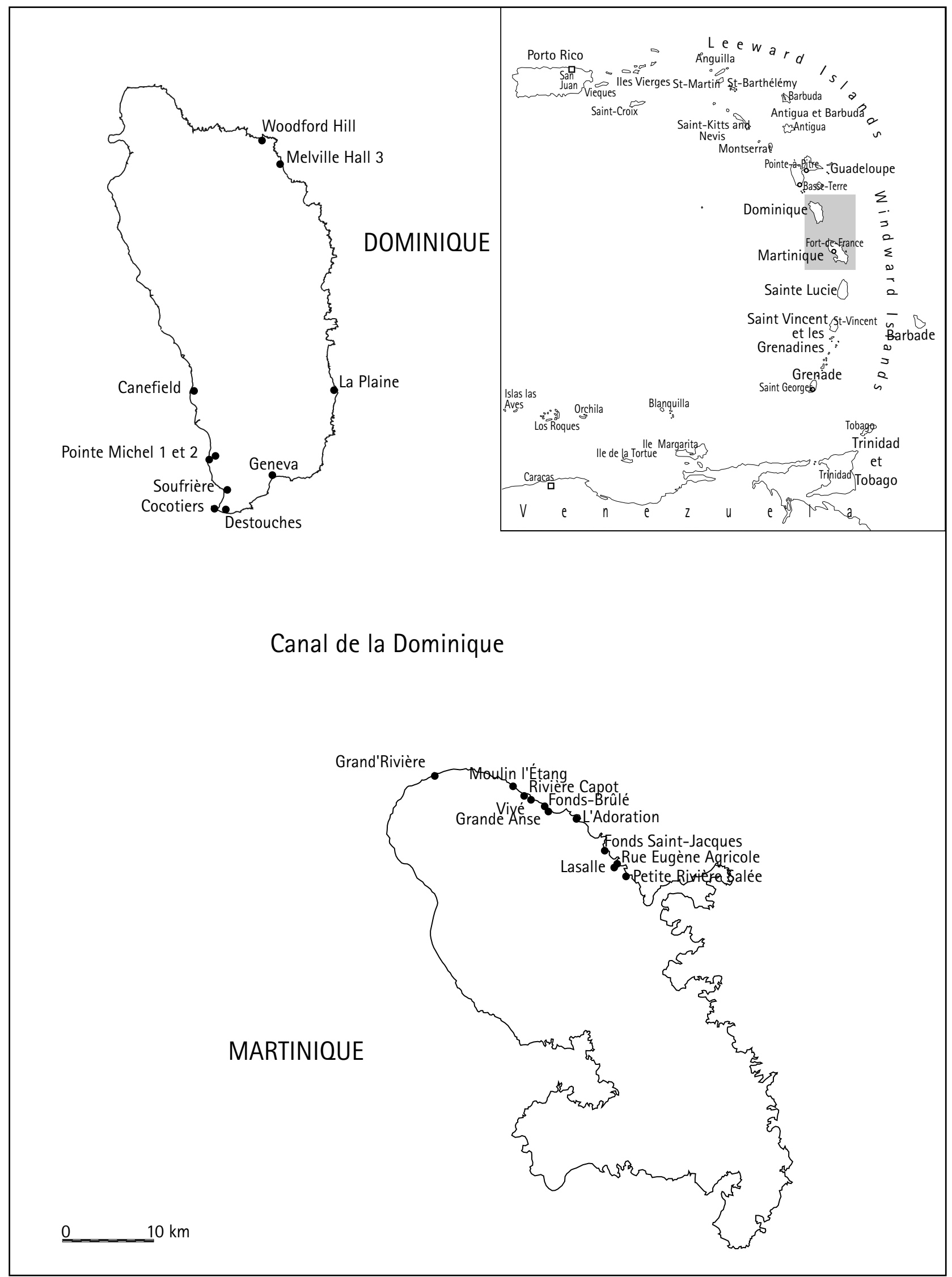

Fig. 1 - Localisation des sites agro-céramistes anciens de Martinique et de Dominique. 


\section{Benoît Bérard | La mission archéologique française en Dominique}

comme R. Myers tentèrent de prouver la faiblesse des arguments avancés (Myers 1978), constitua pendant longtemps un frein considérable au développement de l'archéologie précolombienne en Dominique. Il nous faut être reconnaissant à H. Petitjean Roget d'avoir été le seul à prouver sur le terrain l'inexactitude de cette hypothèse. En 1976 et en 1977, une prospection systématique lui permit de découvrir vingt-quatre nouveaux gisements et il réalisa aussi une petite fouille du site saladoïde cedrosan ancien de Soufrière (Petitjean Roget 1978). La dernière contribution fut le travail de prospection et de cartographie archéologique présenté par L. Honychurch dans la première partie de sa thèse de doctorat (Honychurch 1997). Ainsi, située entre la Martinique et la Guadeloupe qui ont connu un développement de l'archéologie sans commune mesure, la Dominique constituait une sorte de terra incognita.

Pour ce qui est de la protection et de la gestion du patrimoine archéologique précolombien ou historique, la Dominique ne possède aucune législation spécifique. La situation est globalement la même au niveau légal dans les différents pays de l'OECS. En revanche, dans ces différents États, la situation varie fortement du fait de la présence d'associations de protection du patrimoine, plus ou moins anciennes et actives, dont certaines remplissent une mission de service public reconnue par un mandat légal. Elles sont les principaux interlocuteurs des archéologues étrangers souhaitant travailler dans ces pays. En Dominique, au niveau gouvernemental, la recherche est "traditionnellement" gérée par le ministère de l'Éducation. La seule association de protection du patrimoine, SHAPE, se consacre essentiellement à la protection du patrimoine architectural historique. De fait, l'essentiel des activités dans le domaine de l'archéologie est assuré par un ethno-historien dominiquais, le $\mathrm{D}^{\mathrm{r}} \mathrm{L}$. Honychurch.

La mission archéologique Sud-Dominique

La mission archéologique "Sud-Dominique» a donc été d'emblée confrontée à une absence d'interlocuteur institutionnel et à un cadre législatif inexistant : comment obtenir des autorisations de fouilles? Quel serait le statut des collections issues de ces travaux?

Organisation de la mission Le montage de la mission a débuté en 2004, un an avant son démarrage effectif. Ce travail préparatoire nous a permis d'identifier un partenaire local fiable, l'University of the West Indies (UwI), représentée localement par L. Honychurch, et d'établir, en accord avec ce partenaire, les règles de notre intervention dans l'île. L'accord concernait entre autres, le statut des collections archéologiques issues de nos fouilles et nous autorisait à les sortir du pays pendant un an afin de pouvoir les étudier en Martinique. Quant aux autorisations de fouille ou de prospection, elles ont été négociées directement auprès des propriétaires des terrains concernés. Ensuite, il a fallu réfléchir au montage de l'équipe de fouille. En dehors des archéologues de l'INRAP, largement occupés par leur mission première, l'archéologie française dans les Antilles est représentée par de rares chercheurs plus ou moins isolés. Deux stratégies peuvent alors être adoptées pour trouver les compétences nécessaires : faire appel à des collègues français spécialistes d'autres aires géographiques mais acceptant de participer à un projet dans les Antilles facilite beaucoup de choses mais nécessite, de leur part, un investissement soutenu et une réelle volonté de formation au contexte local. Dans ces conditions, il n'est pas toujours facile de trouver des candidats. La deuxième solution consiste à faire appel à des collègues étrangers spécialistes du domaine antillais. Nous avons choisi de mêler ces deux approches pour constituer l'équipe de la mission qui a regroupé JeanPierre Giraud (CRA Languedoc-Roussillon), James Petersen et John Crock (University of Vermont), Joshua Toney (Florida University), Lennox Honychurch (UwI), moi-même de l'université des Antilles et de la Guyane (UAG), ainsi que des étudiants de ces différents établissements et d'autres universités de France métropolitaine. Cet échange culturel entre archéologues français et archéologues des États-Unis a constitué un des éléments les plus enrichissants de notre expérience. Il a permis aux deux parties de mettre en perspective les résultats publiés au cours des dernières années grâce à une meilleure compréhension née d'une pratique quotidienne sur le terrain.

Le programme

L'objectif de nos travaux en Dominique était l'étude des premières occupations agro-céramiques de l'île. L'introduction de la céramique dans les Antilles est liée à la migration de populations originaires du bassin de l'Orénoque au cours de la deuxième moitié du premier millénaire avant notre ère. Ces groupes sont associés à deux ensembles regroupés au sein de la série saladoïde, les sous-séries cedrosanes et huecanes. Après plusieurs années de recherche sur ce sujet en Martinique (Bérard 2004) notre objectif principal en Dominique était de réfléchir à la notion de territoire culturel chez ces groupes. En effet, selon le schéma mis en place par I. Rouse pour l'occupation amérindienne des Antilles, les unités culturelles élémentaires (les complexes) sont rattachées, dans les Petites Antilles, à un espace géographique qui correspond toujours à une île; ce schéma est contraire à celui qu'il a développé dans les Grandes Antilles, où il a défini des territoires culturels centrés sur les canaux qui séparent les îles. Nous voulions tester cette hypothèse qui n'avait jamais véritablement été validée et voir s'il n'était pas possible de définir des territoires composés, mêlant espaces terrestres et maritimes, et intégrant en partie ou en totalité des îles voisines. Il s'agissait de quitter une approche terrestre et insulaire pour tenter de réintégrer l'occupation amérindienne des Petites Antilles dans un cadre plus maritime et archipélique.

Dans ce cas particulier, l'enjeu était de pouvoir évaluer l'importance des relations ayant pu exister entre la Martinique et la Dominique, en particulier entre le nord de la Martinique et le sud de la Dominique. En effet, l'ensemble des occupations saladoïdes cedrosanes anciennes de Martinique connues à ce jour sont localisées sur la côte nord atlantique de l'île, face à la Dominique qui n'est distante que d'une quarantaine de kilomètres. Notre objectif était donc d'étudier les sites contemporains en Dominique, d'autant que les travaux de H. Petitjean Roget avaient démontré que certains d'entre eux se trouvaient dans la partie sud de l'île. 


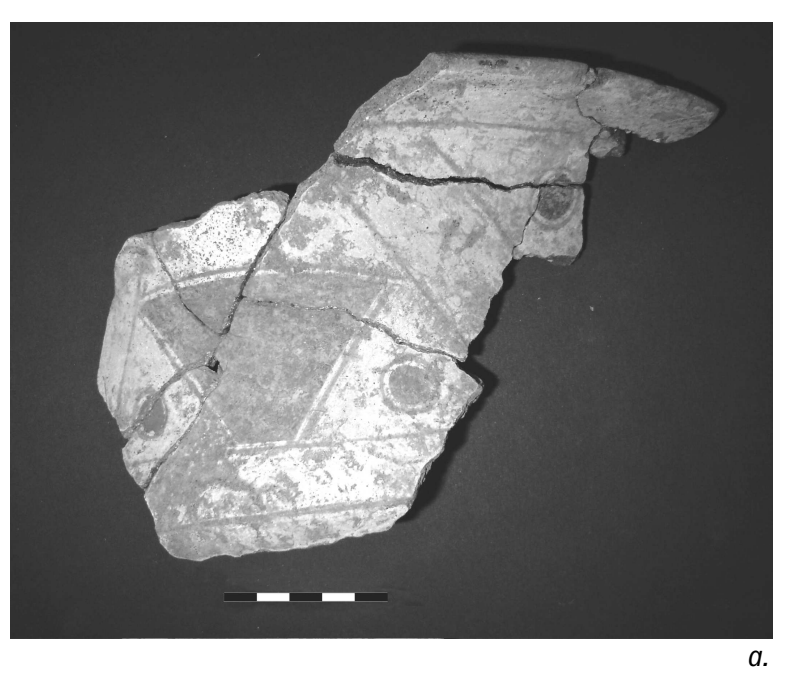

Fig. 2 - Exemple

de la grande proximité

existant entre les productions

saladoïdes cedrosanes anciennes

du sud de la Dominique

et du nord de la Martinique.

$a$-Soufrière, Sud-Dominique ;

$b$ - Vivé, Nord-Martinique.

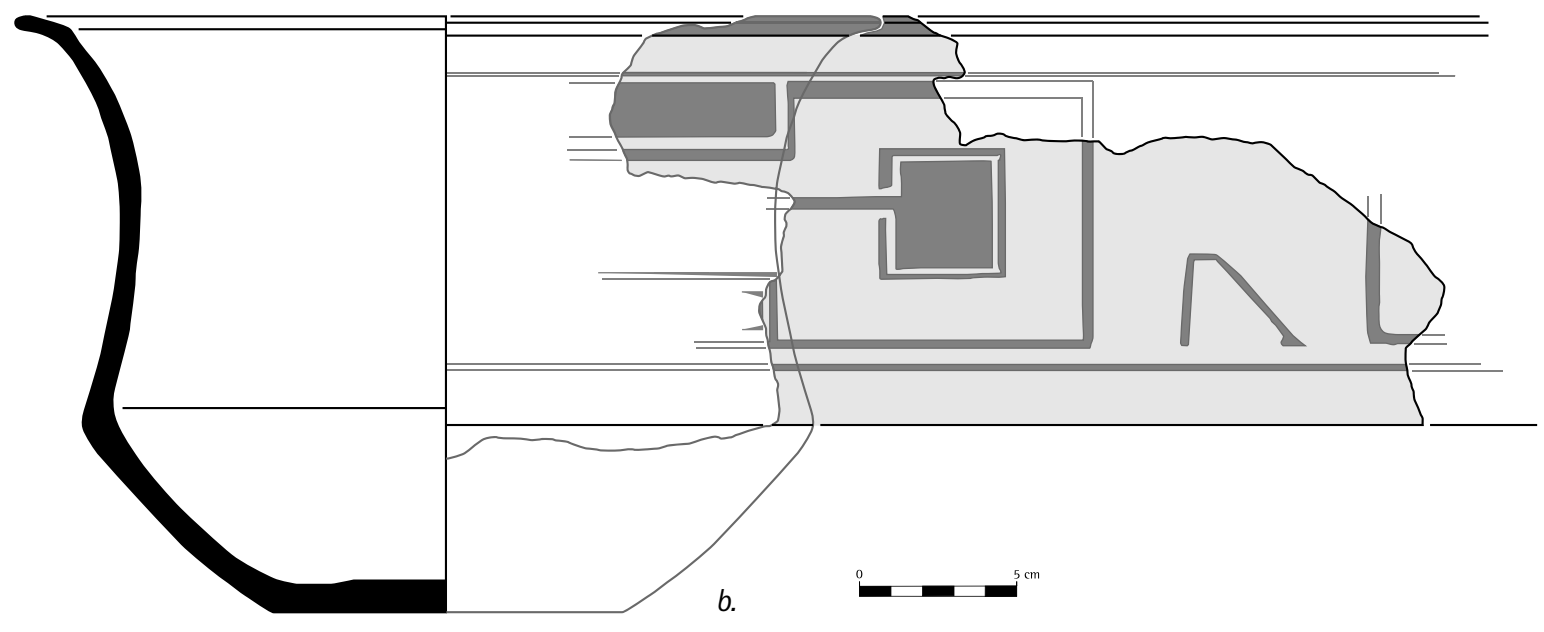

Le choix de cette phase chronologique particulière nous a été dicté par le caractère exceptionnel de la production céramique de ces groupes. En effet, la céramique saladoïde cedrosane ancienne est caractérisée par une grande qualité technique, une importante variété des formes et, surtout, un très fort investissement décoratif (près de $40 \%$ des tessons sont porteurs d'un décors). Associée à d'autres types de données, elle nous semblait donc être un marqueur suffisamment fin pour servir de support à l'étude que nous nous proposions de mener. Notre travail, assez comparable à celui conduit entre 1996 et 2003 en Martinique, s'est articulé autour de quatre axes: un programme de prospection systématique, le sondage de quatre sites agro-céramiques anciens, la réalisation d'une petite fouille sur le site de Soufrière et l'étude de toutes des collections anciennes rattachées à cette phase chronologique. La prospection systématique a augmenté de 40 \% le nombre des sites amérindiens connus dans l'île. Nous avons ainsi pu identifier huit sites agro-céramiques anciens. $\mathrm{Au}$ sein de cet ensemble, les gisements de Pointe Mulâtre, Canefield, Grand Bay et Woodford Hill ont fait l'objet de sondages, mais, malheureusement, les sites de Pointe Mulâtre,
Canefield et Woodford Hill n'ont pas livré de matériel archéologique en place. Les sondages nous ont cependant permis de préciser le contexte stratigraphique des collections anciennes provenant de ces gisements. En fait, les données les plus intéressantes ont été obtenues lors de la fouille du site de Soufrière, conduite en 2005 et en 2006.

\section{Les résultats}

Les données issues de la prospection, des sondages et de la fouille du site de Soufrière sont encore en cours d'analyse. Il est cependant possible de réaliser un premier bilan au niveau scientifique de nos trois campagnes de terrain en Dominique.

Les données paléo-géographiques Les huit sites agro-céramiques anciens aujourd'hui connus en Dominique se divisent en deux ensembles géographiques, un ensemble sud comprenant six gisements (Canefield, Pointe Michel, Soufrière, Destouches, Geneva et La Plaine) et un ensemble nord comprenant deux gisements (Woodford Hill et Melville Hall 3) (fig. 1). L'étude paléo-géographique de ces 


\section{Benoît Bérard | La mission archéologique française en Dominique}

différents lieux d'installation visait à déterminer les caractéristiques qui étaient à l'origine de leur sélection par des groupes arrivant dans une île vierge (ou quasiment vierge) de toute population. Il apparaît tout d'abord que les gisements de l'ensemble sud possèdent des caractéristiques bio-géographiques différentes de celles des sites de l'ensemble nord, chaque ensemble présentant une remarquable homogénéité.

Ainsi, les sites méridionaux sont proches de la mer (moins de $400 \mathrm{~m}$ ) sans être directement sur le rivage, et proches d'une source d'eau douce pérenne (moins de $200 \mathrm{~m}$ ). Par ailleurs, ils reçoivent entre 1270 et $3810 \mathrm{~mm}$ de pluie par an et sont associés à un couvert végétal de type forêt côtière et forêt sempervirente saisonnière tropicale. Enfin, tous ces villages ont été installés sur des sols volcaniques jeunes qui sont les terres agricoles les plus riches de l'île (Lang 1967). Toutes ces caractéristiques sont identiques à celles des sites martiniquais contemporains (Bérard 2004).

Les deux sites de l'ensemble nord sont installés sur des latosols kaoliniques de moindre qualité. Situés dans la zone la moins montagneuse de la Dominique, ils reçoivent entre 1270 et $2540 \mathrm{~mm}$ de précipitations par an. Comme les gisements de l'ensemble sud, ils sont localisés à proximité immédiate d'une rivière pérenne, mais, contrairement à eux, ils sont installés directement sur le rivage et ont d'ailleurs été en partie détruits par le recul de la côte aux cours des derniers siècles.

La céramique

Cette distinction bio-géographique semble confirmée par l'étude céramique.

Nous avons étudié en trois ans plus de 5000 tessons provenant des gisements de l'ensemble sud. Nous avons tout d'abord observé la grande homogénéité de cette production dans les différents sites. Ensuite, il nous a été possible de démontrer l'existence d'une très grande similitude entre ces artefacts et ceux qui ont été découverts dans les sites saladoïdes cedrosans anciens du nord de la Martinique (fig. 2 et 3) (Bérard à paraître).

Malheureusement, Woodford Hill et Melville Hall 3 n'ont livré que des séries céramiques très limitées, issues de sondages et de ramassages de surface. Leur analyse est en cours, mais il apparaît clairement que ces deux sites doivent être rattachés, comme les précédents, aux premières occupations agrocéramistes de l'Arc antillais. Dans les deux collections, des récipients archéologiquement complets ont pu être reconstitués. Or, leur morphologie n'a pas d'équivalent dans les collections provenant des sites du sud de l'île ou du nord de la Martinique.

Ces premières observations semblent donc aller dans le même sens que les constations faites à propos de l'analyse des lieux d'implantation des sites.

Hypothèses et perspectives Si nous avons achevé les opérations de terrain en Dominique, le travail d'analyse quant à lui ne fait que commencer. Les éléments que nous venons de présenter sont le fruit d'études préliminaires et demandent à être confirmés, et surtout affinés, par des analyses plus approfondies. Cependant, une première tendance semble pouvoir être dégagée. Les sites agro-céramistes anciens dominiquais constituent deux ensembles clairement distincts du point de vue géographique. De plus, si au sein de chaque ensemble les caractères environnementaux sont comparables, des différences écologiques distinguent les deux groupes. Cette observation paraît corroborée par l'étude de la céramique. Enfin, les sites du sud de la Dominique paraissent, à tous les niveaux, proches de ceux du nord de la Martinique.

$\mathrm{Si}$ ces premières observations sont confirmées par les résultats des études en cours, il semblerait que nous soyons sur le point de délimiter un territoire culturel, au sein de l'ensemble saladoïde cedrosan ancien, centré sur le canal de la Dominique et intégrant le nord de la Martinique et de sud de la Dominique. Cependant, afin de borner cet ensemble au

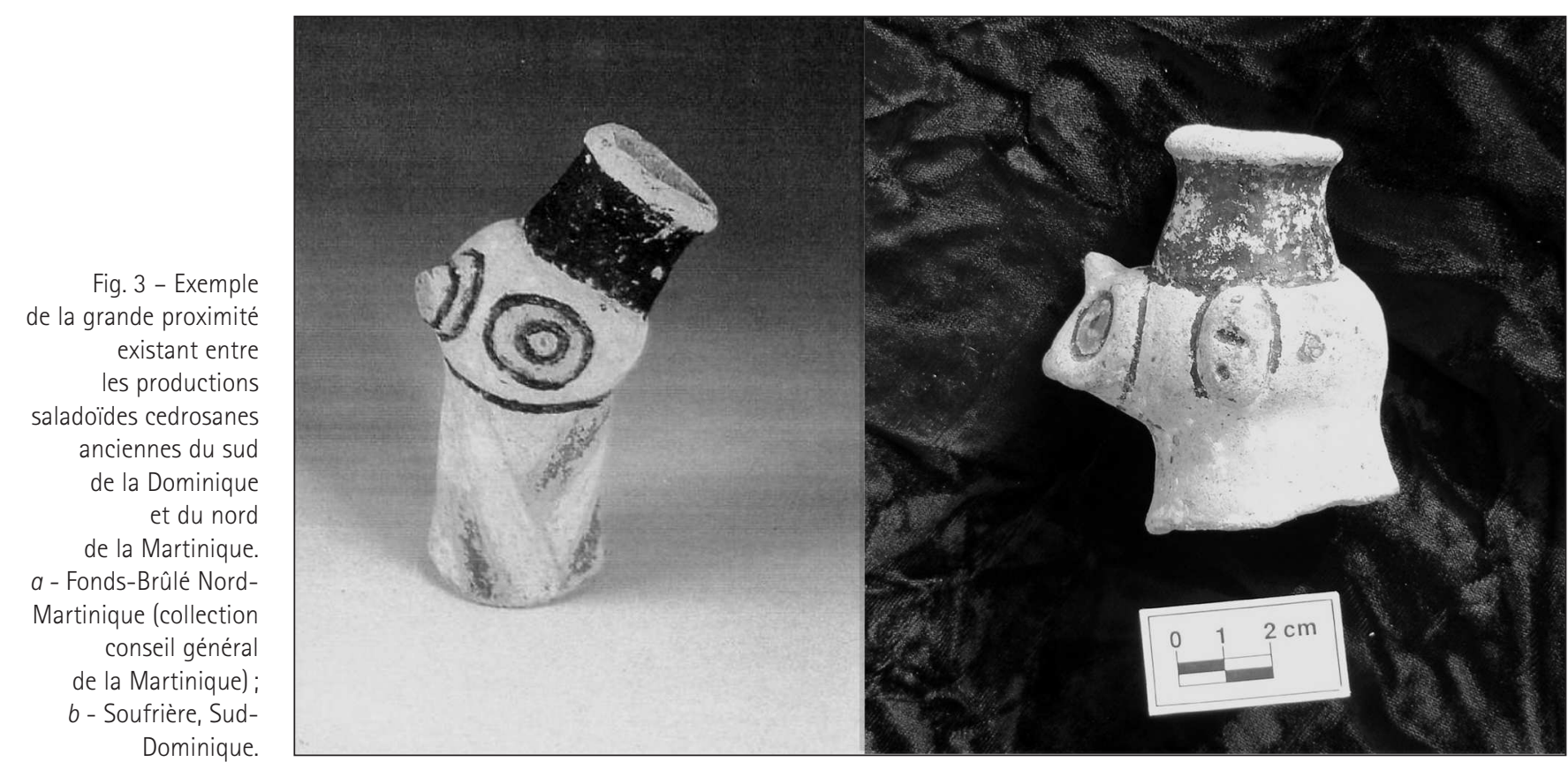




\section{Dossier Des mers de glace à la Terre de Feu}

sud, il faudra dans les années à venir étudier les gisements agro-céramistes anciens de Sainte-Lucie. De même, il serait intéressant de tenter d'établir un lien entre les sites de l'ensemble nord de la Dominique et les gisements contemporains de l'archipel de la Guadeloupe.

Ces premiers résultats ainsi que les perspectives de recherches qui en découlent montrent tout l'intérêt qu'il peut y avoir, pour les archéologues, à se dégager de la perspective insulaire pour inscrire leurs recherches dans un espace plus vaste, celui de l'archipel antillais. Il apparaît ainsi scientifiquement nécessaire pour les archéologues français de sortir de leur pré carré martiniquo-guadeloupéen (certains l'ont d'ailleurs déjà parfaitement compris). Cette nécessité scientifique ne peut avoir comme conséquence que le développement de la recherche française dans les autres îles de l'archipel, et tout particulièrement dans les Petites Antilles. Cependant, afin que cette présence ait un effet réellement bénéfique pour les populations locales, il faut qu'en parallèle il y ait une prise de conscience réelle de l'importance de ce patrimoine par les différentes nations concernées. Dans ces deux domaines, la mise en place d'une bonne gestion du patrimoine archéologique par les États des Petites Antilles et le développement de la recherche française dans la zone, différentes avancées peuvent être soulignées.

Une véritable protection du patrimoine archéologique nécessite à la fois la formation de personnels, l'adoption d'un cadre légal et la mise en évidence de l'intérêt social et économique de ce patrimoine. Pour ce qui est de la formation, plusieurs programmes ont été développés au cours des dernières années ou sont en train d'être mis en place : recrutement d'enseignants d'archéologie à l'université des Antilles et de la Guyane (UAG) comme à l'University of West Indies (UwI) (campus de la Barbade), licence professionnelle "Développement et Protection du patrimoine culturel" à l'UAG, École caribéenne du patrimoine, projet de master international « Patrimoine de la Caraïbe" par l'UAG, Uwi et la London Metropolitan University. Concernant la mise en place d'un cadre légal et la mise en évidence de l'intérêt social et économique de ce patrimoine, il faut souligner l'action de l'ambassade de France auprès des pays de l'Oecs. Elle a ainsi organisé en 2006 un colloque intitulé "Patrimoine et Citoyenneté : pour une harmonisation des politiques patrimoniales dans les pays de la Caraïbe orientale", d'où ont émergé deux projets : la création d'une association régionale destinée à mener une action de lobbying auprès des États de l'Oecs en faveur d'une meilleure gestion de leur patrimoine, et le développement d'un STRABon Caraïbe, sur le modèle de celui développé par la Maison des sciences de l'homme pour le Bassin méditerranéen.

Quant à une l'implication plus soutenue de l'archéologie française dans la Caraïbe, plusieurs éléments doivent être soulignés. Premièrement, quatre thèses sur l'archéologie antillaise ont été soutenues en France au cours de la dernière décennie, et deux autres sont en cours. Ces nouveaux docteurs ont depuis été recrutés comme maîtres de conférences ou ingénieurs à l'INRAP et ils continuent donc à travailler dans la zone. Deuxièmement, au cours de ces dernières années, s'est constituée au sein de l'UmR 8096 "Archéologie des Amériques" du
CNRS une cellule "Antilles» renforcée, après la signature d'une convention avec le ministère de la Culture, par l'intégration en son sein de conservateurs et d'ingénieurs travaillant dans les SRA des départements français d'Amérique. Enfin, il faut souligner les interventions de plus en plus fréquentes de l'INRAP en Martinique et surtout en Guadeloupe. Ses ingénieurs constituent aujourd'hui un important réservoir d'archéologues professionnels et leur expertise, associée à celle des SRA dans le domaine de la gestion du patrimoine archéologique, pourrait jouer un rôle majeur dans la mise en place de programme de coopération internationale visant à aider les États des Antilles à protéger, étudier et mettre en valeur leur patrimoine.

Ainsi, si la mission "Sud-Dominique" a été le premier programme pluriannuel soutenu par le ministère des Affaires étrangères dans les Antilles, tout laisse à penser que de nombreuses autres opérations devraient voir le jour au cours des années à venir.

\section{Bibliographie}

BÉRARD, B. 2004. Les premièresoccupations agricoles del'Arcantillais, Migrations et insularité. Oxford, Archaeopress (BAR International serie 1299, Paris monographs in american archaeology 15, E. Taladoire ed.).

BÉRARD, B. à paraître. "The "South-Dominica" archaeological mission: the Soufrière siten, in: Proceedings of the XXIIth congress of the International Association for Caribbean Archaeology, Kingston, Jamaica, july 23-27 2007.

Evans, C. 1968. "The lack of archeology in Dominica", in: Proceedings of the second International Congress for the study of pre-columbian Cultures in the Lesser Antilles, St. Ann's Garrison, Barbados, July 2428 1967. Barbade, Barbados Museum : 93-102.

FEwKES, J. W. 1922. "A Prehistoric Island Culture Area of America. A Report on Prehistoric Objects from the West Indies in the Museum of the American Indian (Heye Foundation) in 1914", Annual report of the Bureau of American Ethnology, 1913-1914, 34 : 35-281.

HONYCHURCH, L. 1995[1975]. The Dominica Story - A History of the Island. Londres, Macmillan.

HonyCHURCH, L. 1997. Carib to Creole: A History of Contact and Culture Exchange. Thèse non publiée, Hugh College, University of Oxford.

Lang, D. M. 1967. Soils and Land Use Surveys. Trinidad, University of the West Indies (Soil Survey 21).

McKusick, M. B. 1960. Distribution of Ceramic Styles in the Lesser Antilles, West Indies. Ph.D. Thesis, Department of Anthropology, Yale University (University Microfilms, Ann Arbor/London).

MYERS, R. A. 1978. "Ethnohistorical vs. ecological considerations: the case of Dominica's Amerindians", in: J. Benolst et F. M. MeYeR (dir.), Compte-rendu des communications du septième congrès international d'études des civilisations précolombiennes des Petites Antilles, 1116 juillet 1977, Universidad Central de Venezuela, Caracas. Montréal, Centre de recherches caraïbes de l'université de Montréal.

Petitjean Roget, H. 1978. "Reconnaissance archéologique à l'île de la Dominique (West Indies) ", in : J. Benoist et F. M. MeYeR (dir.), Compterendu des communications du septième congrès international d'études des civilisations précolombiennes des Petites Antilles, 11-16 juillet 1977, Universidad Central de Venezuela, Caracas. Montréal, Centre de recherches caraïbes de l'université de Montréal : 81-97.

RODRIGUEZ-LOUBET, F. 1995. "Urbanisation historique du littoral caribéen : Saint-Pierre et la Havane», in: Bilan scientifique de la région Martinique 1994. Trinité, Martinique, Ministère de la Culture et de la Communication, Service régional de l'archéologie : 31. 